\title{
Automatic Illustration of Short Texts via Web Images
}

\author{
Sandro Aldo Aramini, Edoardo Ardizzone and Giuseppe Mazzola \\ Dipartimento di Ingegneria Chimica, Gestionale, Informatica, Meccanica (DICGIM) \\ Università degli Studi di Palermo, Viale delle Scienze bd.6, 90128, Palermo, Italy
}

\begin{abstract}
Keywords: Automatic Illustration, Story Picturing, Semantic Similarity, Semantic Space, Text Analysis, Web Image Search, Google Image.

Abstract: In this paper we propose a totally unsupervised and automatic illustration method, which aims to find onto the Web a set of images to illustrate the content of an input short text. The text is modelled as a semantic space and a set of relevant keywords is extracted. We compare and discuss different methods to create semantic representations by keyword extraction. Keywords are used to query Google Image Search engine for a list of relevant images. We also extract information from the Web pages that include the retrieved images, to create an Image Semantic Space, which is compared to the Text Semantic Space in order to rank the list of retrieved images. Tests showed that our method achieves very good results, which overcome those obtained by using a state-of-the-art application. Furthermore we developed a Web tool to test our system and evaluate results within the Internet community.
\end{abstract}

\section{INTRODUCTION}

In recent years the growing integration between digital cameras and portable devices (mobile phones, PDAs or tablets) has dramatically increased the number of pictures available on the Web. New Internet services, e.g. Flickr, Panoramio, Smugmug, Picasa, were offered to the users as online storage services for their photos, creating huge databases of pictures, that implicitly contain an immense amount of information. These online collections of images consist not only of raw pictures, but also of tags, annotations and descriptions. Each photo individually provides a low information content, but if we consider the whole content of the online collections we are able to extract useful information for different purposes. The key concept is that the desired content is already available onto the Web and we need just to retrieve, filter and organize it. In this way every single photo, tag or comment uploaded by a user on the Web becomes automatically a new information resource, and can be potentially exploited by any user who needs it.

The aim of this work is to exploit the resources of the $\mathrm{Web}$ (photos, descriptions, text) for automatic text illustration, i.e. finding pictures which best illustrate the content of a text. Our system works without any supervising human intervention, and avoids the use of personal annotated database.
When users look for images on the Web by using image search engines (e.g. Google Images), they type one or more keywords in a provided space, and then they have to select the most relevant images from the retrieved list. Our goal is some steps beyond. Our automatic text illustration method needs as input only the text to be illustrated, and gives as output a ranked list of images representing the content of the whole text. Therefore users do not need to choose the proper keywords to create the query to the Image Search Engine, nor to manually select the most relevant images from the retrieved list of images.

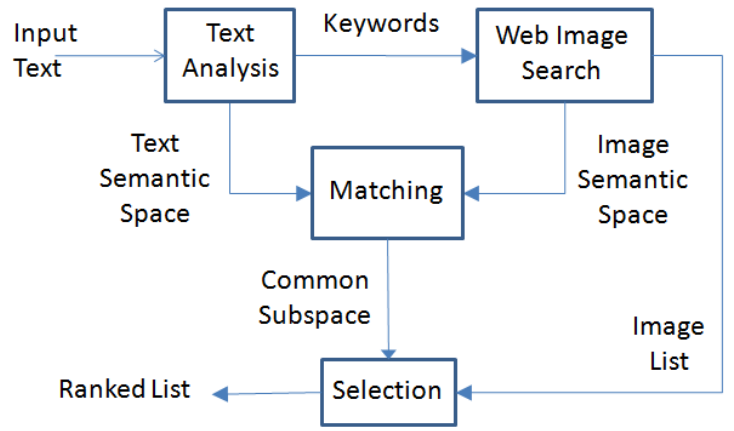

Figure 1: Overall scheme of the proposed approach.

The main contribution of this paper is the exploitation of the Web, as a knowledge base, avoiding the use of a predefined annotated image dataset. In our 
system images are automatically retrieved by using a Web image search engine, dynamically at each query, and annotated with the information extracted from the related Web pages. Our method is able to represent all the possible concepts that can be described by a text, as it is not limited by the use of a personal "ad hoc" image dataset. Furthermore it does not need any time-consuming manual annotation process.

\section{RELATED WORKS}

"Every picture tells a story". This phrase dates back to the beginning of the twentieth century and like the other one, "a picture is worth 1000 words", suggests us the importance of the visual communication through pictures. The relationship between words and pictures has been widely investigated in the past decades. Many efforts have been spent in automatic annotation of images, the process of finding automatically textual tags representing an image (Barnard et al., 2003; Cameiro et al., 2007; Feng et al., 2004; Monay and Gatica Perez, 2007). In this paper we focused on the dual problem, automatic illustration, i.e. the process of finding images which can summarize the content of a text. Studies in recent years have shown that depictive pictures aid learning of texts (Carney and Levin, 2002). Drawings, pictures or illustrations that today we can find in books, short stories or newspapers, facilitate and speed up reader's understanding. However, choosing the right picture to tell a story or to introduce a concept can be a difficult task, involving personal interpretation of the author.

Since automatic illustration is a process involving different research areas, there are a lot of related works into the scientific community. The understanding of a natural language text is still an open problem, likewise picture understanding (Barnard and Forsyth, 2001). However many techniques have been developed to infer semantic information useful to define a semantic-similarity between documents (Kandola et al., 2003). Typical text analysis techniques include latent semantic analysis (Deerwester et al., 1990), or co-occurrence statistical information (Yutaka and Ishizuka, 2004). Even if some approaches have been proposed (Coyne and Sproat 2001; Zhu et al., 2007; Joshi et al., 2006; Miller, 1990; Feng and Lapata, 2010; Rasiwasia et al., 2010; Coelho and Ribeiro, 2011; Delgado et al., 2010), automatic illustration is a problem far away to have a definitive solution. WordsEye (Coyne and Sproat 2001) is an interesting method for automatically converting text into representative 3D scenes, but it does not focus on natural images. (Zhu et al., 2007) presented a system to create a synthetic image from an input text, as a "collage" of pictures that represent some relevant keywords of the text. The "Story Picturing Engine" (Joshi et al., 2006) is an automatic illustrator that performs text illustrations by using Wordnet (Miller, 1990), within an annotated database, and a mutual reinforcement-based ranking algorithm. (Feng and Lapata, 2010) presented a probabilistic approach for automatic image annotation and text illustration, based on the assumption that images and their co-occurring textual data are generated by mixtures of latent topics. The problem of joint modelling the text and image components of multimedia documents (cross-modal retrieval), has been also studied by (Rasiwasia et al., 2010). (Coelho and Ribeiro, 2011) proposed a method which combines text mining techniques and visual descriptors, within an annotated dataset, to illustrate arbitrary texts. (Delgado et al., 2010) proposed a framework that generates automated multimedia presentations to assist news readers.

\section{SYSTEM OVERVIEW}

The goal of the proposed method is to look for a set of images representing the content of an input text, exploiting the Web knowledge and without any user supervision.

Fig. 1 shows the overall scheme of the proposed method. Input text is processed into the text analysis block, which returns some keywords that summarize the text content, and a model of its semantic meaning (Text Semantic Space). Keywords are used to query to an Image Search Engine for a set of images related to these words (Image List). As well, some more information are extracted from the Web pages which include the retrieved images, and processed in order to create a model of the concepts related to these images (Image Semantic Space). The two spaces are intersected to find the Common Subspace, and the retrieved images are ranked on the base of the words that are into this subspace.

Before describing in detail each step of our method, in the next section we will briefly discuss some theory about Semantic Spaces, which are used to model both text and image content.

\section{SEMANTIC SPACES}

In this section we briefly introduce the notion of 
Semantic Space. According to (Lowe, 2001), "a semantic space model is a way of representing similarity of typical context in a Euclidean space with axes determined by local word co-occurrence counts". The co-occurrence of a target word within a dictionary of $\mathrm{D}$ fixed words corresponds to the word position in a space of dimension $\mathrm{D}$. In this space the word position with respect to the other words expresses the similarity of their meanings in the analyzed context.

A Semantic Space model is a quadruple $<W, \mathrm{~L}$, $\mathrm{S}, \mathrm{R}>$ where:

- $\mathrm{W}$ is the set of basis elements (i.e. words);

- $\mathrm{L}$ is a lexical association function, to map words into the basis;

- $\mathrm{S}$ is a similarity measure between words;

- $\mathrm{R}$ is a transformation that reduces the dimensionality of the semantic space.

In the next sections we will explain how Semantic Spaces are used to represent the text and the image contents.

\section{PROPOSED METHOD}

Text analysis methods are typically designed to study a text within a set of documents (corpus) with similar contents, which often defines a specific domain of knowledge. In our work we focused onto single texts, without any reference corpus of related documents, then we cannot use any other information but the input text. On the other hand, we are not limited to a specific domain of knowledge. The purpose of the Text Analysis module is twofold:

- represent the text content as a Semantic Space model;

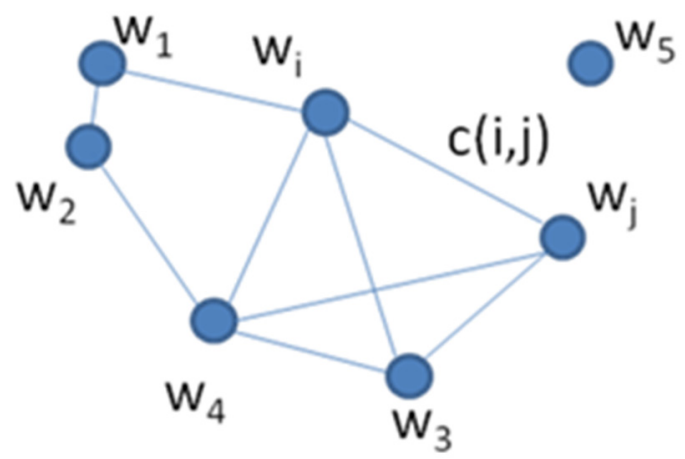

Figure 2: A graph plotting a Semantic Space.
- extract keywords which are representative of the text.

We aim to model the text content in a compact and significant form, as well as to extract relevant keywords. Input text is first scanned to build a dictionary with all its words, and to count related frequencies. The dictionary is filtered to remove common words (articles, prepositions, etc), the "stopwords', which are very frequent in a text, but are not significant to represent its content.

Our system works with two languages (English and Italian) and with two different lists of stopwords. It can be easily extended to other languages, as only a new list of stop-words is needed.

\subsection{Text Semantic Space}

To create a Semantic Space, we divide the input text into sentences. Let $n_{S}$ be the number of sentences in the text. Given a set of $\mathrm{N}$ words $\left(\mathrm{w}_{1}, \mathrm{w}_{2}, . ., \mathrm{w}_{\mathrm{N}}\right)$, we count the number of co-occurrences of the words into the sentences, that is the number of times in which two words are in the same sentence. We focus only on the $\mathrm{N}$ most frequent words in the text, where $\mathrm{N}$ is a value which is supposed to be large enough to capture the text semantic content (see in section 6). We create a $\mathrm{NxN}$ co-occurrence matrix $\mathrm{C}$ in which each term indicates the number of sentences in which the $\mathrm{i}$-th and the $\mathrm{j}$-th co-occur. These terms are divided by the number of sentences $n_{S}$ :

$$
\begin{gathered}
c_{s}(i, j)=\left\{\begin{array}{lll}
1 & \text { if } & w_{i}, w_{j} \in S \\
0 & \text { otherwise }
\end{array}\right. \\
c(i, j)=\frac{\sum_{s=1}^{n_{S}} c_{s}(i, j)}{n_{S}}
\end{gathered}
$$

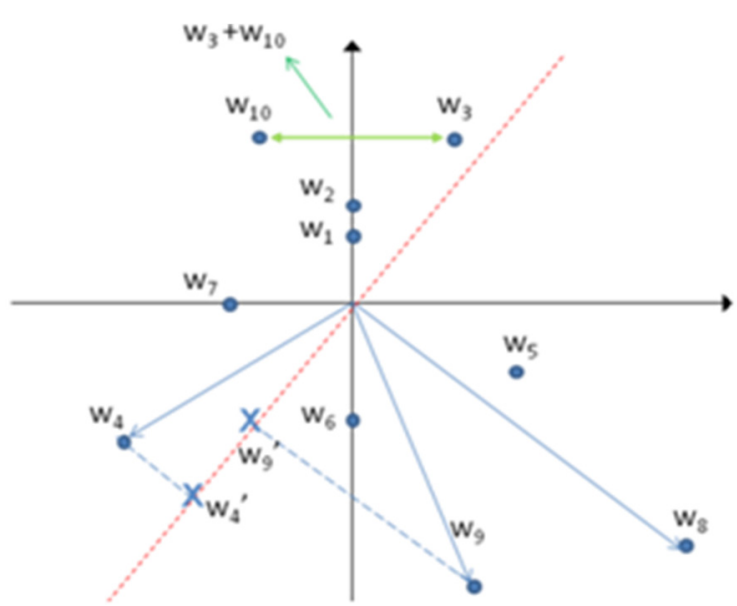

Figure 3: The reduced Text Semantic Space, projected along the principal components axis. 
According to the model presented in section 4:

$-\mathrm{W}_{\mathrm{T}}$ is the set of words in the text;

- $\mathrm{L}_{\mathrm{T}}$ is the identity function, so raw frequencies are used;

- $\mathrm{S}_{\mathrm{T}}$ is the normalized co-occurrence c(i,j) of two words into the sentences of the text;

- $\mathrm{R}_{\mathrm{T}}$ reduces the space dimensionality selecting only the $\mathrm{N}$ most frequent words.

The Text Semantic Space (TSS) can be plotted as a graph (fig. 2), in which the words $\mathrm{w}_{\mathrm{i}}$ are the nodes, while the normalized co-occurrence values c(i,j) are the weights of the edges. In general, the TSS graph may be not completely connected, as some words may occur alone in the sentences.

Note that many text analysis methods use TFIDF (term frequency-inverse document frequency) measure to build a Semantic Space. TF-IDF is a statistical measure that is used to evaluate the importance of a word within a set of documents (corpus). It is proportional to the number of times a word appears in a document, but decreases as the word occurs frequently in the rest of the corpus. We decided to use raw frequencies, rather than TF-IDF, as we do not have a corpus of documents but a single text. Even considering the input text as the whole corpus, and the sentences as the documents to be analyzed, the probability that a (relevant) word occurs more than once in a sentence is negligible, then we decided to use word frequency for our purposes. The choice to work onto a single text is a strictly constraint, but it allows our method to adapt to any type of query and to represent any type of content.

\subsection{Keyword Extraction}

Keyword extraction is a fundamental technique for document summarization and retrieval. If the proper keywords are selected, a reader can easily understand the content of a document and its relationships with the other texts in a corpus of related documents. The goal of this step is to select some words to a query an image search engine, in order to find a list of candidate images that can illustrate the content of the input text. We decided to use two keywords to represent the text content. We noted that using a single keyword would result in a too generic query, as it can represent a too generic concept. Furthermore, if we use a combination of two words, rather than only one, we drastically reduce the problem of polysemy. In fact the second word specifies the meaning of the first one, defining the context of the query. Note that we focused our study onto short texts (e.g. news) as our method aims to find a single image representing the content of the whole text. In fact, in case of longer texts, one single image may be not enough descriptive to represent the content of the whole document. On the other hand, in case of short texts, the use of only two words as keywords may be a tight constraint. Furthermore, with each query, we retrieve such a huge quantity of information from the Web that we can still have, after filtering, enough information for our purposes. The gain of information, with respect to the starting query, will be one of the criteria used during the validation phase of the results.

We also conducted some preliminary experiments using more than two keywords, and we did not note any significant improvement of the results. However, we plan to conduct in the future more experiments, to analyze in depth the influence of the number of keywords on the results.

After having filtered the stop-words from the text, we use five different methods to extract the keywords:

- The two most frequent words (MF);

- The two maximum co-occurring words (MC);

Three methods deriving from the Principal Component Analysis of the normalized co-occurrence (NC) matrix.

The first method simply extracts the two most frequent words in the text. The second one computes the two words that most frequently co-occur in the sentences of the text. The last three methods are inspired to the LSA (Deerwester et al., 1990) approach, and work within the projected text semantic space (PTSS). The PTSS is built applying the Principal Component Analysis to the NC matrix, extracting the two major components, and projecting the columns of $\mathrm{NC}$ (that are the word vectors) onto the principal component space. We decided to work into a 2D-space as, in almost all of our experiments, the cumulative sum of the eigenvalues of the first two eigenvectors is above the $90 \%$, that is a good approximation of the whole energy content. Each word vector is then projected as a point in PTSS (fig. 3), but in some cases we need to further process some peculiar points of the new space. We observed that if two word vectors are projected onto the same point, or are symmetrical to one of the axis or the origin, the corresponding words co-occur in the text always in the same sentences. Therefore they probably are related to the same concept, or are part of a composite word, and are considered as a single word when creating the query. Hence sometimes the final query may be composed by more than two words.

Regarding the geometrical interpretation, the norm of the vectors in PTSS indicates the relevance of the related words within the document (the text). Hence, the word with the largest norm, for us, is the 
most relevant for the input text ( $\mathrm{w}_{8}$ in fig. 3 ). The choice of the second word may be guided by two factors:

- according to the geometrical interpretation, we select the word with the $2^{\text {nd }}$ largest norm (Maximum Norm - MN) (w9 in fig.3);

- we select the word whose vector has the largest orthogonal projection with respect to the direction of the vector with the largest norm (Maximum Orthogonal - MO). It expresses the concept with the maximum semantic distance from the most relevant word (w4 in fig.3).

Note that in most cases the second word extracted by MN and by MO methods is the same. That is not true in general as shown by experimental results in section 6 .

The last method is a combination of MF and $\mathrm{MN}$. We select the two words which maximize the product of the norms of their vectors in PTSS and their normalized frequency into the text (Maximum Weighted Norm - MWN).

\subsection{Image Semantic Space}

The Web Image Search module uses the two keywords from the Text Analysis step to query an Image Search Engine (i.e. Google Image) for a list of images. First, we submit the query to the Web image search engine and we select only the first $M$ valid images, which form our Image List. We use a parser to automatically extract image links from the output HTML Google page. Output links must be validated, as sometimes they refer to images that no longer exist, or that need an authentication to be downloaded. Nevertheless, for efficiency, if we do not get a reply from a link within a short timeout, we discard the link (typically $30 \%$ of the image links are discarded). For each valid image, we also analyze the HTML content of the related webpage. For each webpage we extract metadata keywords, page title and image alternative attribute. To save computational resources, we decided to discard other information (i.e. body text) which should be analyzed with time consuming techniques (e.g.. LSA). Experiments showed that this is a good trade-off between precision and efficiency. Each image is then described by a list of associated tags, which nevertheless includes some terms that are not related to the image content. Tags are filtered discarding those terms that are in a list of stop-words (similarly to text analysis step): articles, conjunctions, adverbs, but also spam, terms typically related to the Web (www, website, blog) or to an image file (photo, gallery) but not to its content. The filtering process significantly reduces the number of words in the tag lists, but it implies that some images will have no tags, and will be discarded as they do not bring any useful information. At last, an image is described by one or more tags, and a word is associated to different images. Hence, we represented this information within a Semantic Space:

- $\mathrm{W}_{\mathrm{I}}$ is the list of all the possible detected words, after filtering;

- $\mathrm{L}_{\mathrm{I}}$ is the number of images associated to each word;

- $\mathrm{S}_{\mathrm{I}}$ is based on the Jaccard coefficient:

$$
S_{I}\left(w_{i}, w_{j}\right)=\frac{\left|I\left(w_{i}\right) \cap I\left(w_{j}\right)\right|}{\| I\left(w_{i}\right) \cup I\left(w_{j}\right)||}
$$

that is the number of images shared by two words $\mathrm{w}_{\mathrm{i}}$ and $\mathrm{w}_{\mathrm{j}}$ divided by the number in the corresponding union set. This metric indicates how much two words are correlated.

- $R_{I}$ is a thresholding process. We select from the basis, as relevant words, those which relates to almost $m=p^{*} M$ images, where $p$ is an input parameter that will be discussed in section 6 , and $M$ is the image dataset size.

The size of the Image Semantic Space (ISS) is not fixed, but changes within every query, as it depends on the number of words which pass the above condition. The Image Semantic Space can be plotted as a graph of the words' correlations, as TSS in fig. 2. For the ISS, nodes consist of words in the basis, reduced by $\mathrm{R}_{\mathrm{I}}$, while edges are weighted by the similarity measure $S_{I}$ (see eq. 2) between words. As in text case, resulting graph may be not completely connected, if a word does not share any image with the other words.

\subsection{Matching and Re-ranking}

The final step of the process is the comparison of the two Semantic Spaces (TSS from the text, and ISS from the Web Image Search), in order to find the set of images in the Image List that better represents the text content.

We intersect the two Semantic Spaces and we select only the words which are shared by the two spaces (fig. 4). Each word in the Common Subspace (CSS), as it is part of ISS, is also associated to one or more images. Nevertheless, each image in the Image List may have one or more tags in this subspace. Our goal is to extract the most relevant common concept from the two semantic spaces, discarding noise. In fact, representing the two spaces as graphs, the best images are those related to the words 


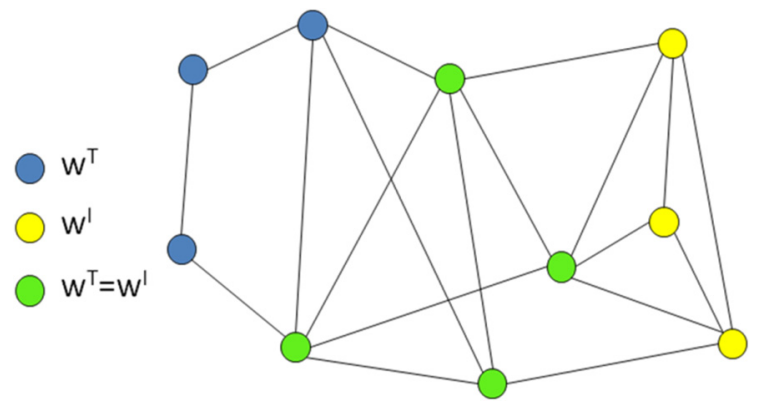

Figure 4: Matching the two Semantic Spaces. Blue nodes are words in TSS, yellow nodes in ISS, green nodes are words shared by the two SS.

into the largest sub-graph of ISS, which are also in CSS. This is not a simple matching of two lists of words. In fact the intersection of the two sub-spaces may result in more than one subgraphs, or may present some isolated nodes. We select all the images in the largest connected component of the intersection and sort them according by the number of tags they have in this subspace. The most significant images are those which have the maximum number of tags associated in CSS, and are presented as output of the system. We can observe that the matching step is mainly based on a graph similarity approach, and then the use of the Semantic Space theory could be overdone. We nevertheless decided to represent the analyzed information by using this theoretical model, as we plan to better exploit this representation in our future works.

\section{EXPERIMENTAL RESULTS}

We implemented two different versions of our system: the first one is a Matlab stand-alone prototype, executed on an Intel Core i7 PC (4 CPU, 1.6 GHz per processor, 4 GB RAM), exploiting the Matlab parallel library to make 4 workers run simultaneously; the second one is web-based version, that had been available online to the Internet community for our tests, implemented with a client side (a simple html page) and a server side (a Java servlet).

\subsection{Stand-alone Prototype}

We tested our prototype on a set of 100 randomly selected news from the Wikinews (link1) archive. To evaluate our system, we used both an objective and a subjective metric. We use as objective metric the semantic similarity of the two spaces, defined as the size of the Common Sub-Space, divided by the number of words in TSS:

$$
S_{C}\left(w_{i}, w_{j}\right)=\frac{|T S S \cap I S S|}{|T S S|}
$$

This is a measure of the semantic similarity of the Text and the Image Semantic Spaces, and it is related to the "gain of knowledge", starting from the initial query. It indicates how many new words have been added to the starting query-words. If the numerator of $\mathrm{S}_{\mathrm{C}}$ is 2 (the lower bound of the intersection), only of the two input keywords are shared by the ISS and the TSS, and the query output may result into a list of images with very different contents. No new knowledge is gained. If $\mathrm{S}_{\mathrm{C}}$ is higher, the ISS shares a larger part of its semantic content with the input text, and it means that new semantic information is added to the two input words.

To help us for a subjective evaluation of the results, we asked 20 persons to test the system. We assigned to each of them 5 short texts from our dataset (texts are typically made of 5-20 sentences) and asked them to read the texts and to evaluate results obtained with all the five proposed keyword extraction methods described in section $5.2(\mathrm{MF}, \mathrm{MC}$, $\mathrm{MN}, \mathrm{MWN}, \mathrm{MO})$. Furthermore we asked them to test the same texts also with a state-of-the-art method, the Story Picturing Engine (Joshi et al., 2006), which has been designed for the same purpose as our application. SPE is an automatic text illustration method, that exploits a personal annotated database of images. A demo was available online when we conducted our tests (link2). We decided to compare the two approaches in terms of final applications, even if the two underlying datasets are different, just because it was difficult to adapt the chosen reference approach to our dataset, which is built dynamically at each query and can be often described by unreliable information (html tags). Moreover, our approach, which has been designed to work using information taken from the Web, should be heavily modified to work within the dataset used by SPE.

We asked testers to indicate if each retrieved image (by our methods and SPE) is Very Relevant (VR), Somewhat Relevant (SR), or Not Relevant (NR) to the text content. Test were performed with $\mathrm{N}=10$ most significant words from the text, $\mathrm{M}=100$ retrieved images, and three values of $p$ for $R_{I}$ (when building ISS): $0.1,0.05,0.03$. SPE online tool has been tested with the granularity value of 0 that, as suggested by authors, must be selected to illustrate the whole story instead of parts of it.

For each input text, and for each proposed keyword extraction method, we measured the similarity Sc and the percentage of VR, SR and NR images into the output list of images (fig.5 shows the 

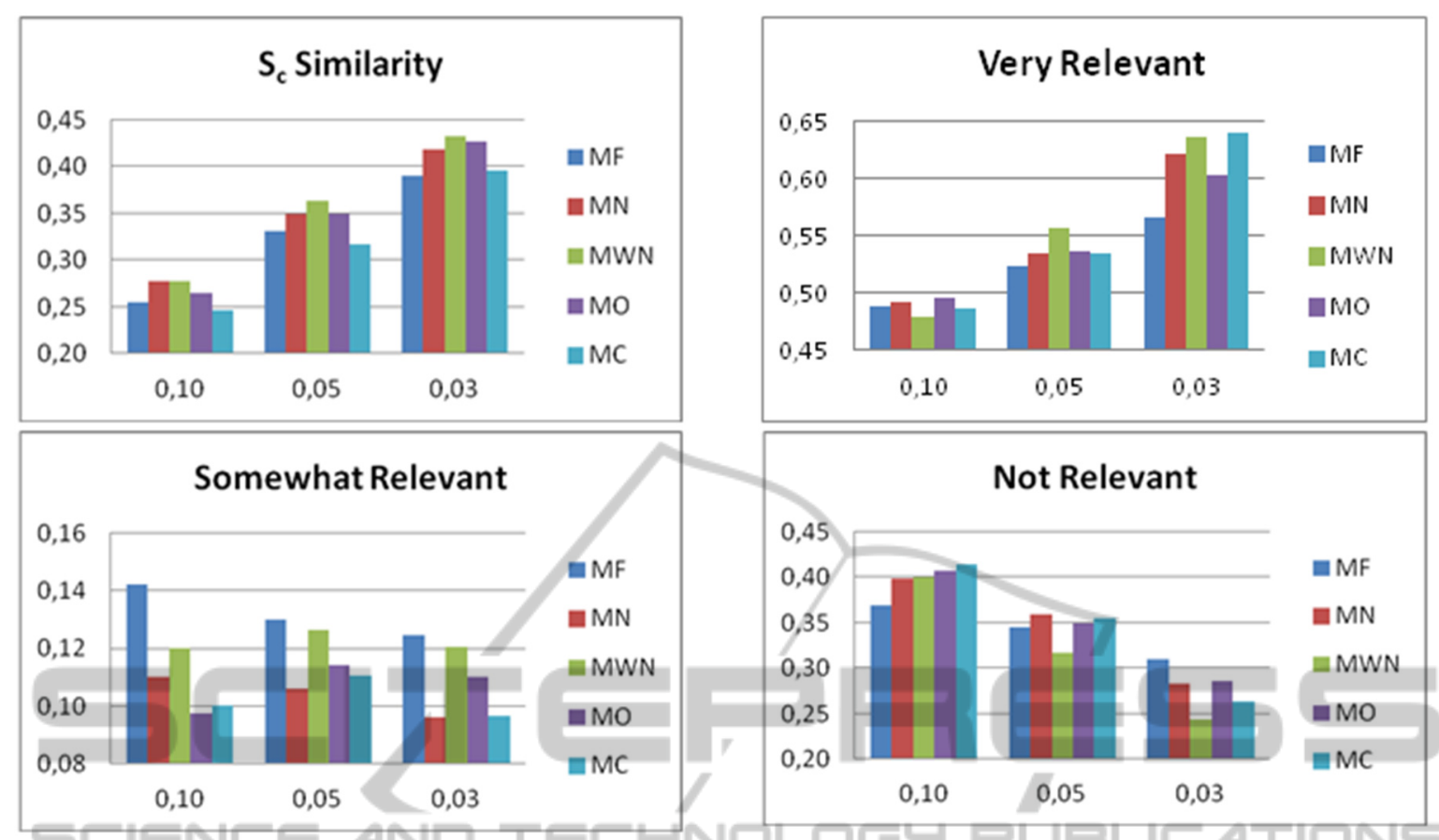

Figure 5: Average values of Sc similarity, and of percentage of VR, SR, NR images, measured for each query, versus the $\mathrm{p}$ parameter, that is related to the threshold used to reduce the Image Semantic Space.

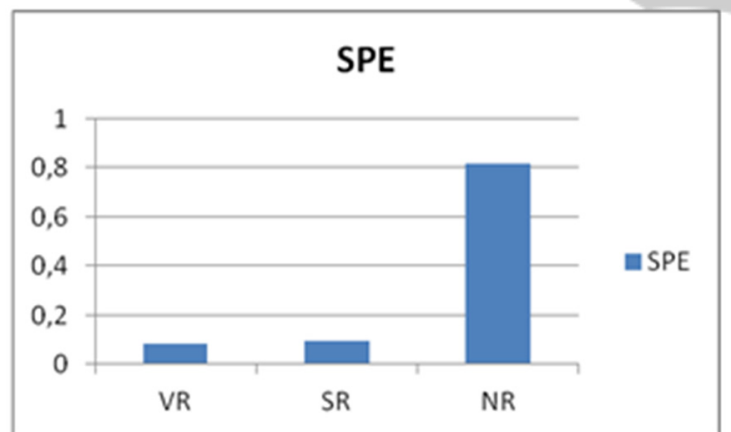

Figure 6: Averaged percentage, per query, of VR, SR and NR images with SPE.

averaged values, per query, within our dataset). Results obtained by using SPE are shown in fig. 6. Fig.7 shows the average number of retrieved images (VR, SR, NR) per query versus the parameter $\mathrm{p}$, that is related to the threshold process described in section 5.3. When reducing the ISS space by using $R_{I}$, the lower the value of $p$, the lower is the minimum number of images that a word, in ISS, must have associated to be considered relevant, and the larger is ISS. Experiments showed that Sc increases when the parameter $p$ decreases as the system works with a larger number of words in ISS, thus increasing the probability to share more words with TSS. Note that we use two keywords to query for images onto the Web, so we expect the minimum number of words

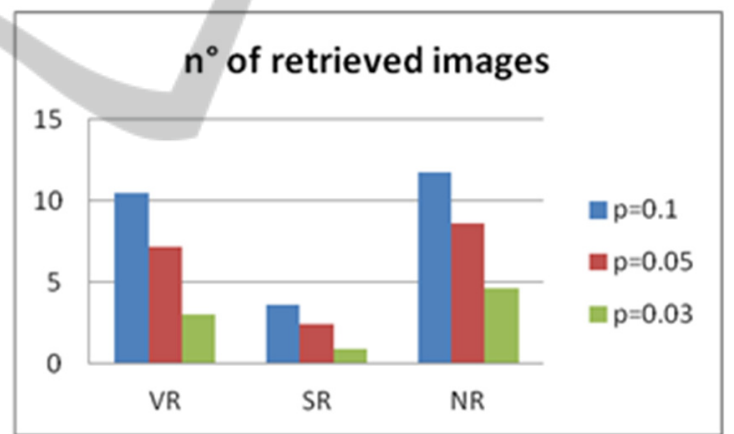

Figure 7: Averaged number of VR, SR and NR retrieved images per query and per all the proposed methods.

in the CSS to be larger or equal than two (the two keywords). When setting $p=0.03$ each image in the output list has, on average, 4 tags, two words more than the two input keywords, gaining a lot of semantic information with respect of the input query. As well, the percentage (VR) of very relevant images per query increases for lower values of $p$. In fact when the two semantic spaces are very similar (Sc is high), the query retrieves a list of relevant images, and the precision increases. With the best configuration (MWN or MC methods, $\mathrm{p}=0.03$ ) we achieved impressive results, more than $63 \%$ of Very Relevant images per query that, if summed with the percentage of SR, grows up to a $75 \%$ of Relevant (VR+SR) images (with MWN method and $p=0.03$ ). On the 
Table 1: Results with the Web Server Application: Semantic Similarity (SS) and average percentage, per query, of relevant (R), not relevant (NR) and not voted (NV) images for the two languages.

\begin{tabular}{|l|l|l|l|l|}
\hline Language & SS & R (\%) & NR (\%) & NV (\%) \\
\hline English & 0.35 & 59 & 33 & 8 \\
\hline Italian & 0.29 & 52 & 22 & 26 \\
\hline
\end{tabular}

other hand, the number of retrieved images decreases with p (fig.7). In fact if ISS and TSS share a high number of words and, as we select only the images which have the maximum number of possible shared tags, the output image list is reduced.

In our tests we observed two typical situations: a "good" illustration gives as output few, but very relevant, images; a "bad" illustration gives as output several and not relevant images. This fact explains why the percentage of VR retrieved images in fig. 7, is different from that in fig. 5. Finally, a low value of $p$ gives the highest precision, but the lowest number of retrieved images.

If comparing our method to Story Picturing Engine (link2), as expected, experiments show that our results overcome those by SPE. Even in the worst case, we achieved a percentage of about $50 \%$ of Very Relevant images per query (in the best case $64 \%$ ), while SPE gives less than $10 \%$ of VR images. Note that SPE is based on a limited personal dataset of images, annotated by authors, then it is able to represent a small set of concepts. On the contrary, our system works extracting the required knowledge from the Web, creating dynamically a new image dataset per each query, and can cover all the possible concepts that can be expressed by a text.

Fig. 8 shows results versus the parameter $p$. Best results are achieved when $p=0.01$. In this case queries typically retrieve few images but with a lot of associated tags (e.g. fig. 8.h shows a single image, but that is very relevant to the input text content). It results in a high precision and a high value of SC.

Regarding efficiency, the whole process takes 23 minutes to be completed. Most of the time is spent validating image links as described in section 5.3 ( $\sim 1$ sec per image, using 4 parallel workers) and downloading metadata $(0,5 \mathrm{sec}$ per URL), then execution time strongly depends on the number of images retrieved by Google Image. Time spent for the other steps of the method is negligible.

\subsection{Web Server Application}

Our Web server application results have been evaluated, as well, using both an objective and a subjec- tive metrics. The objective metric is the semantic similarity defined in eq. 3 . For a subjective evaluation we required some help from the Internet community. We asked to the Web users to test our tool and to leave feedbacks about returned results, selecting relevant and not relevant images. We stored their feedbacks into a database to collect statistical information about query results and their relevancy, Feedbacks helped us to improve the method (e.g. suggesting us new words to add to the list of stopwords), and to evaluate our method performance. We collected more the 1000 queries and we received feedbacks from more or less 300 users.

Results' precision depends on two parameters, described in section 6: the number of images retrieved $\mathrm{M}$ and the minimum number of images $\mathrm{m}$ associated to the tags (which is strictly related to the $p$ value described in section 6.1). We achieved the best results, in terms of speed/precision trade-off, working with $\mathrm{M}=25$ images and $\mathrm{p}=0.04$. Average execution time is about 1 minute per query. Average percentages of precision, per query, are shown in Table 1, for both English and Italian languages. In terms of Semantic Similarity, results show that we gained knowledge with respect of the two input keywords: each output image is tagged with more than the two keywords used to create the query. Precision results show that our method gives as output a very low number of not relevant images, and achieve very good results in terms of relevant images $(60 \%$ circa for English queries, more than $50 \%$ for Italian queries).

\section{CONCLUSIONS}

In our work we faced several problems:

- we worked with a single text, while many text analysis techniques study documents within a corpus of similar documents, which typically specifies the domain of knowledge. We dynamically create the domain at each query, without any external information. In this way we can represent any type of content, and we are not limited to a specific domain.

- we needed to model the text content in order to extract relevant information, which would be compared with those extracted from the Web. Semantic Space models helped us in this step, and we used PCA to reduce space dimensionality.

- we needed significant keywords to query an Image Search Engine for a list of relevant images. We proposed and discussed five different methods to extract keywords from the input text or from its models. 


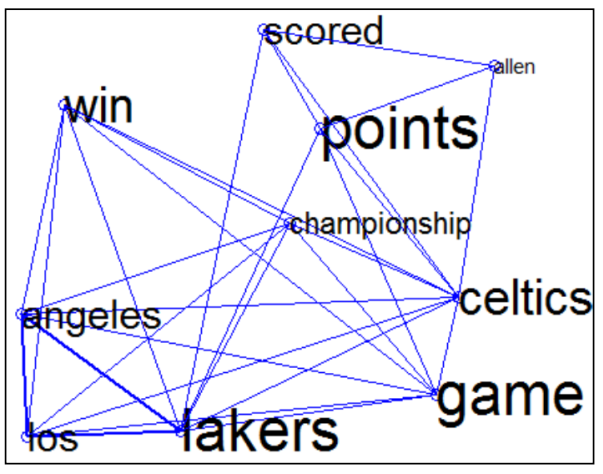

a) TSS

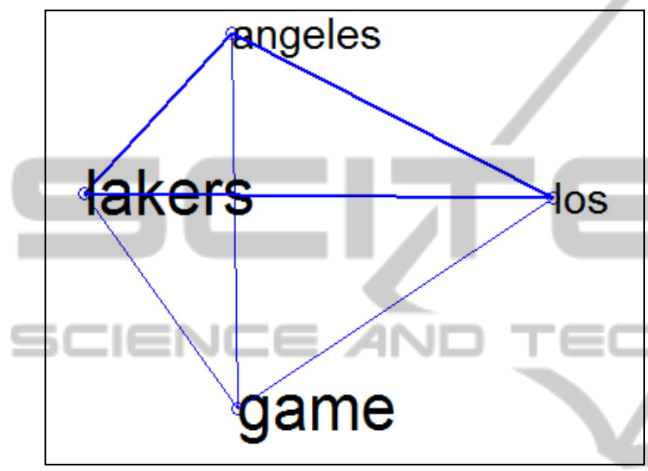

c) CSS, $p=0.1$

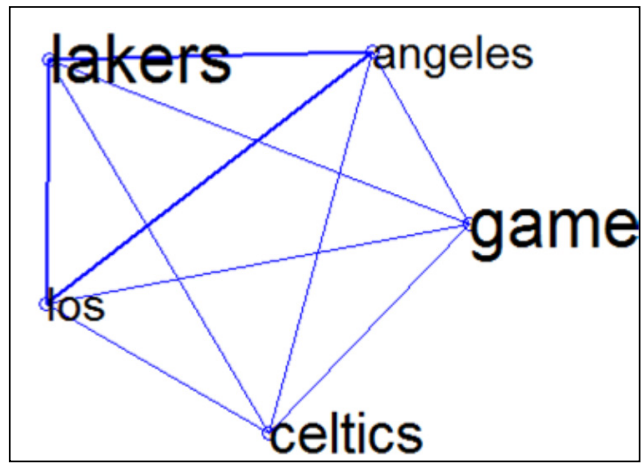

e) CSS, $\mathbf{p}=\mathbf{0 . 0 5}$

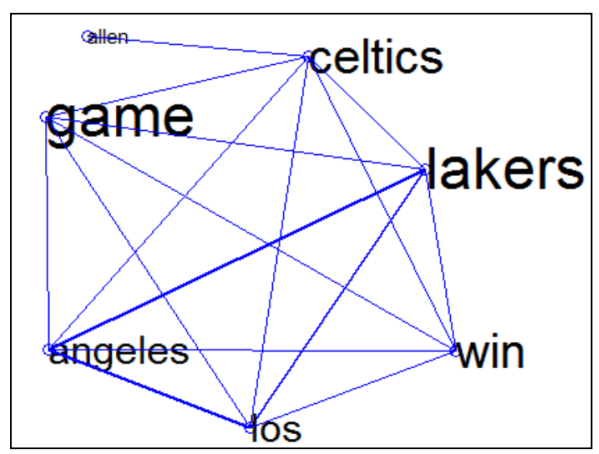

g) CSS, $\mathrm{p}=\mathbf{0 . 0 3}$
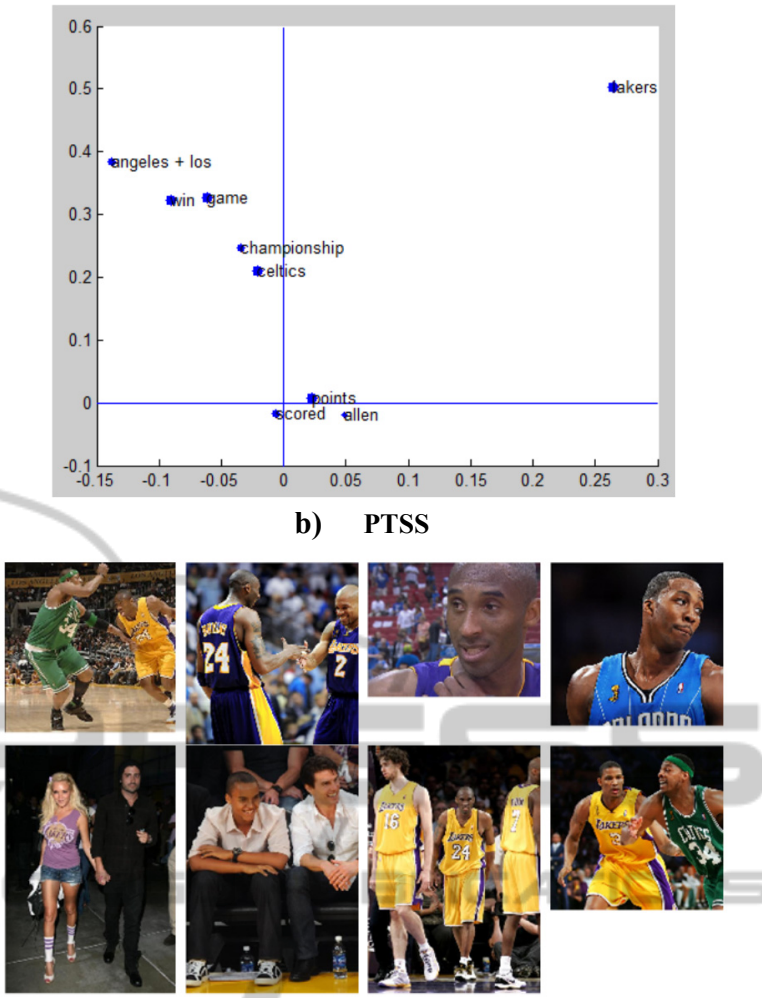

d) Output tags: angeles, lakers, game, los

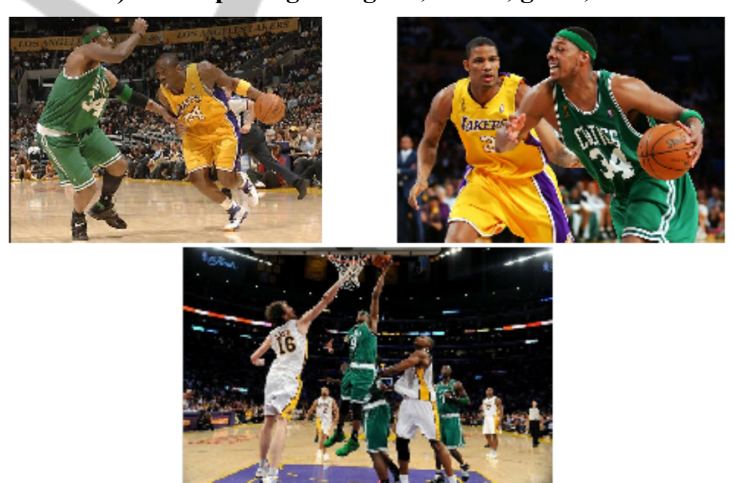

f) Output tags: angeles, celtics lakers, game, los,

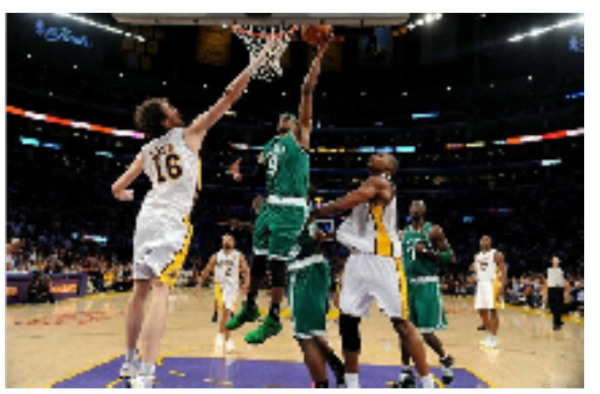

h) Output tags: allen, angeles, celtics, game, lakers, los, win

Figure 8: Results obtained with MWN with different values of p. (9.d shows only part of the output list). Input text at (link3). 
- we had to represent the content of the images of the Web. We extracted information from the Web pages containing the retrieved images, i.e. metadata, and we modelled this content as a Semantic Space, similarly to the text case.

- we needed to compare the two Semantic Space. For that, we defined a similarity measure which indicates how much the two spaces relates to the same semantic meaning.

- we needed to re-rank the list of retrieved images. We propose to extract the words from the common subspace of the two semantic spaces. Images are ranked on the basis of the number of tags they share in the common sub-space.

Results are very satisfactory, and impressive if compared to those obtained with SPE. The main difference between the two methods is the image dataset. SPE uses a personal collection of photos, annotated by hand, hence is very limited by the number of images and by the concepts represented in its dataset. We use Google Image to create, dynamically at each query, a new image dataset to work within. Thus we exploit the knowledge of the Web, increasing the chances to find images relevant to the text content.

Furthermore our method has been designed to be multi-language, as it can be easily extended to other language if the proper list of stop-words is created. We also developed a Web implementation of the proposed method, as a new service to Internet users.

We are confident that our solution will interest news or advertising agencies, newspapers websites, bloggers or in general all the users who search for information into the Web.

At last, the "core" of our system is general purpose, and can be used to compare texts, HTML pages, and all types of annotated document, that can be retrieved from the Web. That is, we can use Google Search (Youtube, Wikipedia, etc.) instead of Google Image, to query for any type of tagged contents which can be useful to describe an input text.

\section{REFERENCES}

Barnard, K., Duygulu, P., et al., 2003. Matching words and pictures. JMLR, 3:1107-1135.

Barnard, K., and Forsyth, D., 2001. Learning the Semantics of Words and Pictures. Proc. International Conference on Computer Vision, pp. II: 408-415, 2001.

Carney, R. N., and Levin, J. R., 2002, "Pictorial illustrations still improve students' learning from text", Educational Psychology Review, 2002, 14(1), 5-26.

Carneiro, G., Chan, A., et al., 2007. Supervised learning of semantic classes for image annotation and retrieval.
IEEE Transactions on Pattern Analysis and Machine Intelligence, 29(3):394-410.

Carney, R. N., and Levin, J. R., 2002. Pictorial illustrations still improve students' learning from text. Educational Psychology Review, 14(1), 5-26.

Coelho, F., and Ribeiro, C., 2011, Automatic illustration with cross-media retrieval in large-scale collections. In Content-Based Multimedia Indexing (CBMI), 2011 9th International Workshop on (pp. 25-30). IEEE.

Coyne, B., and Sproat, R., 2001. Wordseye: An automatic text-to-scene conversion system. In Proceedings of the 28th Annual Conference on Computer Graphics and Interactive Techniques. SIGGRAPH 2001, 487-496.

Deerwester, S., Dumais, S. T., et al., 1990. Indexing by latent semantic analysis. Journal of the American Society For Information Science, 41(6), 391-407.

Delgado, D., Magalhaes, J., \& Correia, N., 2010. Automated illustration of news stories. In Semantic Computing (ICSC), 2010 IEEE Fourth International Conference on (pp. 73-78). IEEE.

Feng, Y., and Lapata, M., 2010. Topic models for image annotation and text illustration. In Proceedings of the NAACL HLT. Association for Computational Linguistics, Los Angeles, California, pages 831-839.

Feng, S., Manmatha, R, and Lavrenko, V., 2004. Multiple bernoulli relevance models for image and video annotation. In CVPR, volume 2(2004), pp. 1002-1009.

Joshi, D., Wang, J .Z., and Li, J., 2006. The story picturing engine - a system for automatic text illustration. ACM Transactions on Mul-timedia Computing, Communications, and Applications, 2(1):68-89.

Kandola, J. S., Shawe-Taylor, J., and Cristianini, N., 2003. Learning semantic similarity, In Neural Information Processing Systems 15 (NIPS 15), pp. 657-664.

Lowe, W., 2001. Towards a theory of semantic space. In Proceedings of the Twenty-Third Annual Conference of the Cognitive Science Society 2001 (pp. 576-581). Mahwah, NJ: Erlbaum.

Miller, G. 1990. WordNet: An on-line lexical database. Int. Journal of Lexicography, Special Issue, 3(4).

Monay, F., and Gatica-Perez, D., 2007. Modeling semantic aspects for cross-media image indexing. IEEE Transactions on Pattern Analysis and Machine Intelligence, 29(10):1802-1817.

Rasiwasia, N., Pereira, J. C., et al. 2010. A new approach to cross-modal multimedia retrieval. In Proceedings of the International Conference on Multimedia (MM '10), 251-260.

Yutaka, M., and Ishizuka, M., 2004. Keyword extraction from a single document using word co-occurrence statistical information. Int'l Journal on Artificial Intelligence Tools, 13(1):157-169.

Zhu, X., Goldberg, A. B., et al., 2007. A text-to-picture synthesis system for augmenting communication. In Proceedings of the 22nd national conference on Artificial intelligence, Vol. 2, 1590-1595 2007.

link1: http://en.wikinews.org/wiki/Main_Page.

link2: http://alipr.com/spe/

link3: http://en.wikinews.org/wiki/Los_Angeles_Lakers need_to_win_game_six_to_tie_NBA_championship. 